\title{
Bayesian Reflectance Component Separation
}

\author{
Ramón Moreno, Manuel Graña ${ }^{\star}$, Alicia d'Anjou, Carmen Hernandez \\ Grupo de Inteligencia computacional, \\ www.ehu.es/ccwintco \\ University of the Basque Country
}

\begin{abstract}
We work on a Bayesian approach to the estimation of the specular component of a color image, based on the Dichromatic Reflection Model (DRM). The separation of diffuse and specular components is important for color image segmentation, to allow the segmentation algorithms to work on the best estimation of the reflectance of the scene. In this work we postulate a prior and likelihood energies that model the reflectance estimation process. Minimization of the posterior energy gives the desired reflectance estimation. The approach includes the illumination color normalization and the computation of a specular free image to test the pure diffuse reflection hypothesis.
\end{abstract}

\section{Introduction}

Works on reflectance map estimation $[17,4,10,9,12,1,2]$ usually need to impose some assumptions like the knowledge of a color segmentation of the image, the detection of color region boundaries or color discontinuities, or the knowledge of the decomposition into linear basis functions of the surface color. The approach presented here does not impose any such assumption and does not need previous segmentations of the image. Most of the works in the literature are based on the Dichromatic Reflection Model (DRM) [8], and we will also follow this model for the development of our approach. We follow a Bayesian approach [3] to model the desired result as constraints implemented in an a priori distribution. We postulate the a priori distribution based on the idea developed in [13] that the derivatives of the logarithmic images of both diffuse image and specular free must be equal in order to have pure diffuse pixels.

Section 2 gives the reflection modelling background, section 3 describes our Bayesian model giving the expressions for the a priori and likelihood energies. Section 4 presents some experimental results. Section 5 gives some summary conclusions and ideas for further work.

\section{Reflection modelling}

The DRM was proposed by Shafer [8]. It describes the surface reflection of light in dielectric materials as the sum of two components, the diffuse and specular

\footnotetext{
* The Spanish Ministerio de Educacion y Ciencia supports this work through grant DPI2006-15346-C03-03
} 
terms. The diffuse reflection component exhibits the color of the material due to different light wavelengths are more or less absorbed as light is scattered by the material. The specular reflection component is essentially determined by the color of incident light. The model of the image taken with a digital camera is as follows

$$
\begin{gathered}
\mathbf{I}(x)=w_{d}(x) \int_{\Omega} S(\lambda, x) E(\lambda) \mathbf{q}(\lambda) d \lambda+w_{s}(x) \int_{\Omega} E(\lambda) \mathbf{q}(\lambda) d \lambda \\
I(x)=w_{d}(x) \mathbf{B}(x)+w_{s}(x) \mathbf{G},
\end{gathered}
$$

where $\mathbf{I}=\left\{I_{r}, I_{g}, I_{b}\right\}$ is the color of an image pixel obtained through a camera sensor, $x=\{x, y\}$ are the two dimensional coordinates of the pixel in the image, $\mathbf{q}=\left\{q_{r}, q_{g}, q_{b}\right\}$ is the three element vector of sensor sensitivity, and $w_{d}(x)$ and $w_{s}(x)$ are the weighting factors for diffuse and specular components, respectively, which depend on the geometric structure at location $x, S(\lambda, x)$ is the diffuse spectral reflectance, $E(\lambda)$ is the illumination spectral power distribution function, which is independent of the spatial location $x$ because we assume a uniform illumination color. The integration is done over the visible light spectrum $\Omega$. We define the following chromatic terms:

- Image chromaticity (normalized RGB space) : $\boldsymbol{\Psi}(x)=\frac{\mathbf{I}(x)}{I_{r}(x)+I_{g}(x)+I_{b}(x)}$

- Diffuse Chromaticity: $\boldsymbol{\Lambda}(x)=\frac{\mathbf{B}(x)}{B_{r}+B_{g}+B_{b}}$

- Specular or Illumination Source Chromaticity: $\boldsymbol{\Gamma}=\frac{\mathbf{G}}{G_{r}+G_{g}+G_{b}}$.

The image can be written in terms of diffuse an specular chromaticity $\mathbf{I}(x)=$ $m_{d}(x) \boldsymbol{\Lambda}(x)+m_{s}(x) \boldsymbol{\Gamma}$, where $m_{d}(x)=w_{d}(x)\left[B_{r}(x)+B_{g}(x)+B_{b}(x)\right]$ and $m_{s}(x)=$ $w_{s}(x)\left(G_{r}+G_{g}+G_{b}\right)$. We can see that the diffuse chromaticity depends on the pixel location $x$, while the specular chromaticity does not, because we assume a uniform illumination color. Both weighting factors depend on the geometric structure at location $x$.

For the ensuing processes, we will be assuming that illumination colors (the specular component) will be pure white, so that $\Gamma_{r}=\Gamma_{g}=\Gamma_{b}$. The illumination corrected image is computed as $\mathbf{I}^{\prime}(x)=\frac{\mathbf{I}(x)}{\Gamma^{e s t}(x)}$. Where $\Gamma^{e s t}$ is the estimation of the illumination color, that can be estimated by some of the methods proposed in the literature $[6,14,15,2]$. The quotient is computed as the Hadamard quotient (applied at each component independently). The normalized image can be expressed as $\mathbf{I}^{\prime}(x)=m_{d}^{\prime}(x) \boldsymbol{\Lambda}^{\prime}(x)+\frac{m_{s}^{\prime}(x)}{3}$, where $\boldsymbol{\Lambda}^{\prime}$ is the illumination color normalized diffuse chromaticity.

It is possible to obtain an specular free image [17] from the color normalized image by the following procedure:

1. Compute at each pixel the minimum of all of its three color bands $\tilde{\mathbf{I}}(x)=$ $\min \left\{I_{r}^{\prime}(x), I_{g}^{\prime}(x), I_{b}^{\prime}(x)\right\}$, therefore $\tilde{\mathbf{I}}(x)=m_{d}^{\prime}(x) \tilde{\mathbf{\Lambda}}(x)+\frac{m_{s}^{\prime}(x)}{3}$, where $\tilde{\mathbf{\Lambda}}(x)=$ $\min \left\{\Lambda_{r}^{\prime}(x), \Lambda_{g}^{\prime}(x), \Lambda_{b}^{\prime}(x)\right\}$. 
2. Compute at each pixel the difference of the normalized image and the one obtained in the previous step $\mathbf{I}^{s f}(x)=\mathbf{I}^{\prime}(x)-\tilde{\mathbf{I}}(x)=m_{d}^{\prime}(x)\left[\boldsymbol{\Lambda}^{\prime}(x)-\tilde{\boldsymbol{\Lambda}}(x)\right]$, so that the specular component dissapears from the image.

\subsection{Separation Method}

We will base our Bayesian model in the key element of the method proposed in $[11,13]$. The pure diffuse pixels can be characterized by the following relation:

$$
\triangle(x)=\operatorname{dlog}\left(\mathbf{I}^{\prime}(x)\right)-\operatorname{dlog}\left(\mathbf{I}^{s f}(x)\right)=0,
$$

where $d \log \left(\mathbf{I}^{s f}(x)\right)=\frac{\partial}{\partial x} \log \left(\mathbf{I}^{s f}(x)\right)$ and $d \log \left(\mathbf{I}^{\prime}(x)\right)=\frac{\partial}{\partial x} \log \left(\mathbf{I}^{\prime}(x)\right)$, the logarithm is computed pixel wise, and the spatial derivative can be computed in several ways, for instance in [13] it is computed on the scalar value image given by the summation of the three channels. It can be easily verified that $d \log \left(\mathbf{I}^{\prime}(x)\right)=\frac{\partial}{\partial x} \log \left(m_{d}^{\prime}(x)\right)=d \log \left(\mathbf{I}^{s f}(x)\right)$ for pure diffuse pixels if the diffuse chromaticity of neighboring pixels is the same. That means that the method works well inside homogenous color regions, and needs the estimation of color region boundaries. When $\triangle(x)>0$ in eq. 3 and the pixel is not at a color boundary and a pure specular pixel, then it has some specular component that can be removed to get the diffuse reflectance component. The method proposed in [13] follows from an heuristic observation about the distribution of pixels in the maximum chromaticity versus (normalized illumination color) intensity space. Non diffuse pixels are decreased in intensity iteratively to search for the pure diffuse pixel value.

\section{Bayesian Modelling}

Given an image $f$ and a desired unknown response of a computational process $d$, Bayesian reasoning gives, as the estimate of $d$, the image wich maximizes the $A$ Posteriori distribution $P(d \mid f) \propto e^{-U(d \mid f)}$, where the A Posteriori energy can be decomposed in to the $A$ Priori $U(d)$ and Likelihood (Conditional) $U(f \mid d)$ energies $U(d \mid f)=U(f \mid d)+U(d)$. The Maximum A Posteriori (MAP) estimate is equivalent minimize the posterior energy function

$$
d^{*}=\arg \min _{d} U(d \mid f)
$$

The Likelihood energy $U(f \mid d)$ measures the cost caused by the discrepancy between the input image $f$ and the solution $d$. The A Priori energy $U(d)$ is a model of the desired solution, usually built as a Random Markov Field (RMF), so that the A Priori energy can be built up as the summation of the local energies at the pixels, which are expressed as summations over the set of cliques including the pixel, weighted by the local potential parameter. A Priori energy usually incorporates any desired constraint, such as smoothness, into the model. 
We will assume a Gaussian Likelihood distribution plus a Chromaticity preservation constraint, therefore the Likelihood energy will have the following expression:

$$
U(d \mid f)=\sum_{i=1}^{m} \frac{\left(f_{i}-d_{i}\right)^{2}}{2 \sigma^{2}}+\sum_{i=1}^{m}\left(\Psi_{i}^{f}-\Psi_{i}^{d}\right)^{2},
$$

where $f_{i}$ and $d_{i}$ are the RGB pixel values a the $i$-th pixel position for the observed and desired image, respectively. Also, $\Psi_{i}^{f}$ and $\Psi_{i}^{d}$ denote the chromaticity pixels of the observed and desired image, respectively.

The A Priori energy is built up from two components. The first one is the Chromaticity continuity:

$$
U_{\Psi}(d)=\sum_{i=1}^{m} \sum_{j \in N_{i}} \sum_{c \in\{r, g, b\}}\left(\Psi_{i, c}^{d}-\Psi_{j, c}^{d}\right)^{2} .
$$

The second modelling the estimation of the derivatives in eq. 3 as the cliques of the RMF. That is, we assume that the local energy at pixel $d_{i}$ is defined as

$$
U_{\triangle}\left(d_{i}\right)=\left(\operatorname{dlog}\left(d_{i}\right)-\operatorname{dlog}\left(d_{i}^{s f}\right)\right)^{2},
$$

where $d_{i}^{s f}$ is the $i$-th pixel of the specular free image, computed as described above, and $\operatorname{dlog}($.$) in means the local estimation of the derivative, which is$ approximated as follows:

$$
d \log \left(d_{i}\right)=\frac{1}{\# N} \sum_{j \in N_{i}} \log \left(\frac{I\left(x_{j}\right)}{I\left(x_{i}\right)}\right),
$$

where $N_{i}$ is the local neighborhood of pixel $d_{i}$, and \#N is its cardinality. After some manipulations, the local derivative component of the A Priori energy is derived as:

$$
U_{\triangle}\left(d_{i}\right)=\left(\sum_{j \in N_{i}} \sum_{c \in\{r, g, b\}} \log \frac{d_{j, c} d_{i, c}^{s f}}{d_{i, c} d_{j, c}^{s f}}\right)^{2} .
$$

This local energy is equivalent to the Kuk-Jin ratio criterion [17]. The derivative component of the A Priori energy is, therefore, the addition of these local energies:

$$
U_{\triangle}(d)=\sum_{i=1}^{m} U\left(d_{i}\right),
$$

and the A Priori energy is given by the addition $U(d)=U_{\triangle}(d)+U_{\Psi}(d)$.

\section{Some Experimental results}

In this section we report some experimental results applying the Bayesian approach described above. The starting value for the energy minimization process 
is set to $f=d(0)=\mathbf{I}^{\prime}$. Each iteration step of the energy minization involves the computation of the specular free image $d^{s f}(t)$ of the current hypothesis $d(t)$ of the optimal estimation $d^{*}$. Instead of using a Monte Carlo minimization technique [3], such as Simulated Annealing, we have employed a simple heuristic to determine the new hypothesis $d(t+1)$, consisting in the reduction of the intensity of the pixels preserving their chromacity components relative ratios. Although simple, this strategy does in fact produce a minimization of the energy function, as can be appreciated in figure 1, where we plot an instance of the energy function evolution. We have tested our approach on some images already tested by some authors in the literature i.e. [13,12] among others. Figure 2 shows the result over a well known test image with two colors and two light sources. Our algorithm does not include any modelling of the underlying color regions in the scene, such as in [12], so it can be appreciated that the almost pure specular pixels can not be corrected, because there almost no chromatic information left in them. To improve our approach we will be including a color map field in the model, to be able to assign those pixels the most likely color. The figure 3 shows a complex geometry image. Our estimation of the diffuse reflectance component recovers the underlying geometry, with some blurring effects.

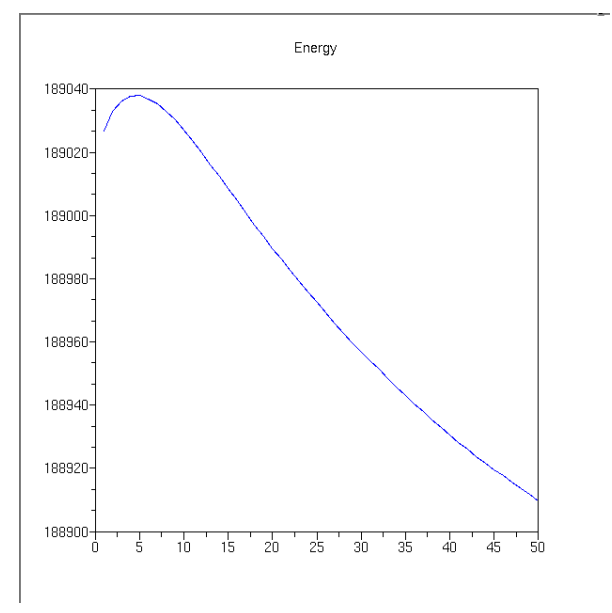

Fig. 1. Evolution of the energy function in an instance run of the algorithm

\section{Conclusions and further works}

We have presented a Bayesian approach to the problem of reflection component separation. As in previous works, our approach works with only one image [13] and does not need any additional assumption, such as models of the colors in scene o previous color segmentations of the image. We compute the specular 


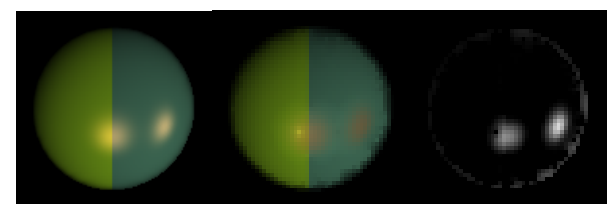

Fig. 2. From left to righ, the original image, the estimated diffuse reflection component, and the estimated especular component

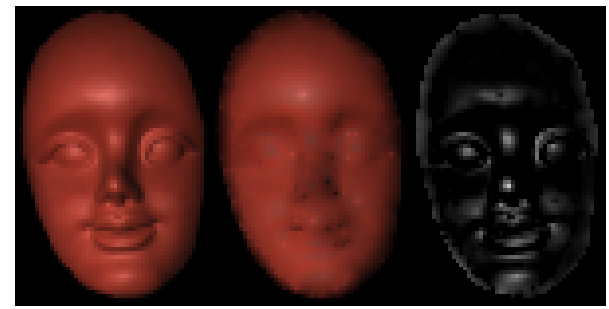

Fig. 3. From left to righ, the original image, the estimated diffuse reflection component, and the estimated especular component

free image, which can be done on the fly for each hypothesis. We have tested the approach applying a simple heuristic to provide new hypothesis from the previous iteration, with quite encouraging results. From the experiments we detect the need to incorporate a color map field in the A Priori model, so that the color of almost purely specular pixels can be recovered more easily. The problem of diverse color illumination sources will be dealt with in further works. We will also extend our works to other imaging models $[7,16,5]$.

\section{References}

1. Wan-Chun Ma Tim Hawkins Pieter Peers Charles-Felix Chabert Malte Weiss Paul Debevec. Rapid acquisition of specular and diffuse normal maps from polarized spherical gradient illumination. In Eurographics Symposium on Rendering $200 \%$. Eurographics Symposium on Rendering 2007, 2007.

2. Zhouyu Fu, R.T. Tan, and T. Caelli. Specular free spectral imaging using orthogonal subspace projection. In Pattern Recognition, 2006. ICPR 2006. 18th International Conference on, volume 1, pages 812-815, 2006.

3. Winkler G. Image analysis, random fields and dynamic Monte Carlo methods. Springer Verlag, 1995.

4. Kenji Hara, Ko Nishino, and Katsushi Ikeuchi. Light source position and reflectance estimation from a single view without the distant illumination assumption. IEEE Trans Pattern Anal Mach Intell, 27(4):493-505, Apr 2005.

5. Henrik Wann Jensen, Stephen R. Marschner, Marc Levoy, and Pat Hanrahan. A practical model for subsurface light transport. In Proceedings of the 28th annual conference on Computer graphics and interactive techniques, pages 511-518. ACM, 2001. 
6. Yoo-Jin Choi Kuk-Jin Yoon and In So Kweon. Illuminant chromaticity estimation using dichromatic slope and dichromatic line space. In Korea-Japan Joint Workshop on Frontiers of Computer Vision, pages 219-224. FCV, 2005.

7. Bui Tuong Phong. Illumination for computer-generated images. PhD thesis, The University of Utah, 1973.

8. Steven A. Shafer. Using color to separate reflection components. Color Research and Aplications, 10:43-51, april 1984.

9. Robby T Tan, Ko Nishino, and Katsushi Ikeuchi. Color constancy through inverseintensity chromaticity space. J Opt Soc Am A Opt Image Sci Vis, 21(3):321-334, Mar 2004.

10. Robby T Tan, Ko Nishino, and Katsushi Ikeuchi. Separating reflection components based on chromaticity and noise analysis. IEEE Trans Pattern Anal Mach Intell, 26(10):1373-1379, Oct 2004.

11. R.T. Tan and K. Ikeuchi. Separating reflection components of textured surfaces using a single image. In Computer Vision, 2003. Proceedings. Ninth IEEE International Conference on, pages 870-877vol.2, 13-16 Oct. 2003.

12. R.T. Tan and K. Ikeuchi. Reflection components decomposition of textured surfaces using linear basis functions. In Computer Vision and Pattern Recognition, 2005. CVPR 2005. IEEE Computer Society Conference on, volume 1, pages 125131vol.1, 20-25 June 2005.

13. R.T. Tan and K. Ikeuchi. Separating reflection components of textured surfaces using a single image. Pattern Analysis and Machine Intelligence, IEEE Transactions on, 25(2):178-193, 2005.

14. Javier Toro. Dichromatic illumination estimation without pre-segmentation. Pattern Recogn. Lett., 29:871-877, 2008.

15. Javier Toro and Brian Funt. A multilinear constraint on dichromatic planes for illumination estimation. IEEE transactions on image processing : a publication of the IEEE Signal Processing Society, 16:92-7, 2007. PMID: 17283768.

16. Gregory J. Ward. Measuring and modeling anisotropic reflection. SIGGRAPH Comput. Graph., 26:265-272, 1992.

17. Kuk-Jin Yoon, Yoojin Choi, and In So Kweon. Fast separation of reflection components using a specularity-invariant image representation. In Image Processing, 2006 IEEE International Conference on, pages 973-976, 8-11 Oct. 2006. 